\title{
Flutter instability prediction techniques for bridge deck sections
}

\author{
I. Robertson, S.J. Sherwin*, P.W. Bearman \\ Department of Aeronautics, \\ Imperial College of Science, Technology and Medicine, \\ Prince Consort Road, London, SW7 $2 B Y, U . K$.
}

\begin{abstract}
SUMMARY
In order to investigate the fluid/structure interaction of a bridge deck in a cross wind, a two dimensional $h p /$ Spectral fluid solver has been modified to incorporate a body undergoing translational and rotational motion. A moving frame of reference is attached to the body to utilise the efficiency of a fixed mesh solver. The critical reduced velocity at which a bridge deck undergoes a two degree of freedom flutter instability is then predicted using various methods: a theoretical linear potential model; quasi-steady theory; a linear evaluation of applied forces using prescribed motion; and free translational and rotational motion of the structure. These predictions are compared with experimental data and the various merits of each scheme are reported. Copyright (C) 2000 John Wiley \& Sons, Ltd.
\end{abstract}

KEY WORDS: bridge deck; instability; $h p /$ Spectral elements; CFD; flutter; fluid/structure interaction

\section{INTRODUCTION}

The possibility of fluid structure interaction leading to catastrophic failure should never be underestimated, particularly in long-span bridge design. This was illustrated by the Tacoma Narrows Bridge disaster in 1940. The construction of a modern long-span bridge can cost in excess of $£ 250$ million and new design strategies using lighter materials and improved construction techniques are continually being developed. Whilst these advances may appear to be economical the savings must be considered alongside the inherent stability and safety of the design. One major risk is in the area of fluid structure interaction leading to aerodynamic instability. Currently, a detailed understanding of why one design is stable to a higher windspeed than a second seemingly almost identical design is lacking.

The aerodynamic stability of bridge decks is established by carrying out numerous wind tunnel tests using spring-mounted sectional models that reproduce, at small scale, the relevant geometric and structural characteristics of the full scale bridge. Many experiments are required to determine the critical wind speed for the onset of instability, each with slightly different

${ }^{*}$ Correspondence to: S.J. Sherwin, Department of Aeronautics, Imperial College, Prince Consort Road, London, SW7 2BY UK. e-mail: s.sherwin@ic.ac.uk

Contract/grant sponsor: EPSRC; contract/grant number: GR/L48096

Copyright (C) 2000 John Wiley \& Sons, Ltd.

Received

Revised 
model characteristics, until an acceptable solution is found. A particularly sensitive aspect of the design is the leading edge region of a bridge deck. Computational fluid dynamics (CFD) has the potential to provide the engineer cheaply, quickly and efficiently with the information required to ensure safe but economical designs. It is unlikely in the short term that CFD will replace wind tunnel testing but it is possible that it could play a role at an early stage in a test program to eliminate unsatisfactory designs and thus shorten the route to finding an acceptable bridge configuration. CFD can also provide insights into fluid structure interactions that may be very difficult, or even impossible, to obtain from conventional wind tunnel experiments.

Over the last decade various CFD algorithms have been developed to address these needs. Among them are finite element methods [1, 2, 3], based on the ALE formulation; finite volume methods [4], using deformable hybrid grids; mesh free discrete vortex methods [5, 6]; and spectral element methods [7], using coordinate transformation. The majority of these cases are confined to the study of the body with transverse or in-line motion and mainly concentrate on lock-in $[1,2,4,8,9,10]$, where the structure is excited by von Karman vortex shedding and there is interaction between the vortex and vibration frequencies. Comparatively little work has been done on the torsional fluid structure interaction. However, it is this instability that was responsible for the failure of the Tacoma Narrows Bridge [11, 12, 13].

In this paper the motion of a single body structure undergoing rotational and translational motion due to a cross wind is simulated. The critical reduced velocity for the onset of flutter is numerically predicted and compared with other analytical, semi-analytical and experimental methods.

In section 2 the aerodynamic and structural governing equations are specified and in section 3 the bridge deck geometry and governing parameters are given. In section 4 prediction of the critical reduced velocity is performed using various methods and the results compared with experimentally generated data.

\section{SOLUTION METHOD}

For the problem of an elastically mounted rigid body acted upon by flow forces, the governing equations are the incompressible Navier-Stokes equations with moving boundary conditions, i.e.,

$$
\begin{gathered}
\nabla \cdot \mathbf{v}=0 \\
\frac{\partial \mathbf{v}}{\partial t}+(\mathbf{v} \cdot \nabla) \mathbf{v}=-\frac{1}{\rho} \nabla p+\nu \nabla^{2} \mathbf{v}, \quad \text { in } D(t) \\
\mathbf{v}=\mathbf{v}_{b}(t) \quad \text { on } \Gamma(t)=\partial D(t)
\end{gathered}
$$

where $\mathbf{v}$ is the fluid velocity, $p$ is the pressure and $\Gamma(t)$ denotes the interface between the flow and the body and moves with the unknown velocity $\mathbf{v}_{b}$.

For a single rigid body, its planar motion can be described in terms of three displacement components defined at the centre of gravity [14], $\mathbf{X}=(\zeta, \eta, \theta)^{T}$, where $\zeta$ and $\eta$ are the translational displacement components in the $x$ and $y$ direction, respectively, and $\theta$ denotes the rotational displacement component. We may write the governing equation for this planar motion as

$$
\mathbf{M} \ddot{\mathbf{X}}+\mathbf{D} \dot{\mathbf{X}}+\mathbf{K X}=\overline{\mathbf{F}},
$$

Int. J. Numer. Meth. Fluids 2000; 00:1-6 
where $\mathbf{M}, \mathbf{D}$ and $\mathbf{K}$ are the mass, damping and stiffness matrices, respectively, $\overline{\mathbf{F}}$ is a vector whose components are external forces and moment and a dot above a variable denotes differentiation with respect to time. The mounting system for the body is assumed to have both stiffness and damping.

Instead of directly solving the coupled system of equations (1) to (4), an efficient strategy is to solve the Navier-Stokes equations and structural equation explicitly and thereby decouple their solutions on each time step. Therefore, at each time level, we initially solve the NavierStokes equations to obtain the aerodynamic force and moment acting on the body, the force and moment are then used as an input into the structural solver to predict the displacement of the body at the next time level. This displacement is then employed to determine the flow domain (precisely the interface of fluid and structure) in the fluid solver.

The most general and widely used method to simulate moving boundary problems is the Arbitrary Lagrangian-Eulerian formulation (ALE) where the computational mesh at the farfield boundaries is stationary and the mesh on the moving boundary takes the same velocity as the structure [14]. The configuration of the computational mesh therefore changes at each timestep resulting in the Navier-Stokes solver constantly being regenerated, negating the ability to utilise efficient direct solvers for static meshes.

In [10], a numerical method is formulated which enables efficient computations by evaluating the governing two dimensional aerodynamic equations (1-3) on a fixed mesh. These equations are calculated in a moving frame of reference which is fixed to the structure and the relationship between this moving frame of reference and a stationary fixed frame of reference is established which can be treated as additional forcing terms in the Navier-Stokes equation (2). This extra computational cost is small compared to that saved by evaluating the equations on a static mesh.

\subsection{Model equations}

We will consider the non-dimensional form of the Navier-Stokes equations

$$
\begin{gathered}
\nabla^{*} \cdot \mathbf{v}^{*}=0 \\
\frac{\partial \mathbf{v}^{*}}{\partial t^{*}}+\left(\mathbf{v}^{*} \cdot \nabla^{*}\right) \mathbf{v}^{*}=-\nabla^{*} p^{*}+R e^{-1}\left(\nabla^{*}\right)^{2} \mathbf{v}^{*} .
\end{gathered}
$$

The variables in the equations above have been non-dimensionalised as follows,

$$
\begin{gathered}
t^{*}=\frac{t U}{D}, \quad x^{*}=\frac{x}{D}, \quad y^{*}=\frac{y}{D}, \\
u^{*}=\frac{u}{U}, \quad v^{*}=\frac{v}{U}, \quad p^{*}=\frac{p}{\rho U^{2}}, \\
\operatorname{Re}=\frac{\rho U D}{\mu}=\frac{U D}{\nu},
\end{gathered}
$$

where $D$ is a characteristic length of the body, and $\rho$ and $U$ are the density and the freestream velocity respectively. Correspondingly, the forces and moment are non-dimensionalised by,

$$
F_{x}^{*}=\frac{F_{x}}{\rho D U^{2}}, \quad F_{y}^{*}=\frac{F_{y}}{\rho D U^{2}}, \quad M_{\theta}^{*}=\frac{M_{\theta}}{\rho D^{2} U^{2}} .
$$

The governing structural equation for the one degree of freedom heaving mode is

$$
m \ddot{y}+c_{y} \dot{y}+k_{y} y=F_{y},
$$


where $m$ is the mass per unit length of the body, $c_{y}$ is the damping constant, $k_{y}$ is the stiffness constant, and $y$ denotes the transverse location of the centre of the body. If we use the same non-dimensional scales as applied to the Navier-Stokes equations, then we obtain

$$
\begin{gathered}
\ddot{y}^{*}+2 \xi_{y}\left(\frac{2 \pi}{U_{y}}\right) \dot{y}^{*}+\left(\frac{2 \pi}{U_{y}}\right)^{2} y^{*}=\frac{F_{y}^{*}}{n_{y}} \\
U_{y}=\frac{U}{f_{y} D} \quad(\text { reduced velocity }), \quad n_{y}=\frac{m}{\rho D^{2}} \quad \text { ( mass ratio ). }
\end{gathered}
$$

Here $f_{y}$ is the natural frequency of the structure in the vertical direction and $\xi_{y}$ is the structural damping ratio. For the one degree of freedom torsional mode, equation (4) becomes

$$
I \ddot{\theta}+c_{\theta} \dot{\theta}+k_{\theta} \theta=M_{\theta},
$$

where $I$ is the moment of inertia, $c_{\theta}$ is the torsional damping constant, $k_{\theta}$ is the torsional stiffness constant, and $\theta$ denotes the rotational angle of the body around the elastic centre. The right-hand side of equation (12) is the moment relative to the elastic centre. The nondimensional form of (12) is

$$
\ddot{\theta}+2 \xi_{\theta}\left(\frac{2 \pi}{U_{\theta}}\right) \dot{\theta}+\left(\frac{2 \pi}{U_{\theta}}\right)^{2} \theta=\frac{M_{\theta}}{n_{\theta}},
$$

where

$$
\left.\left.U_{\theta}=\frac{U}{f_{\theta} D} \text { ( reduced velocity }\right), n_{\theta}=\frac{I}{\rho D^{4}} \text { ( moment of inertia ratio }\right) .
$$

In the following sections the asterix notation to denote non-dimensional variables will be relaxed and all variables should be taken as non-dimensional.

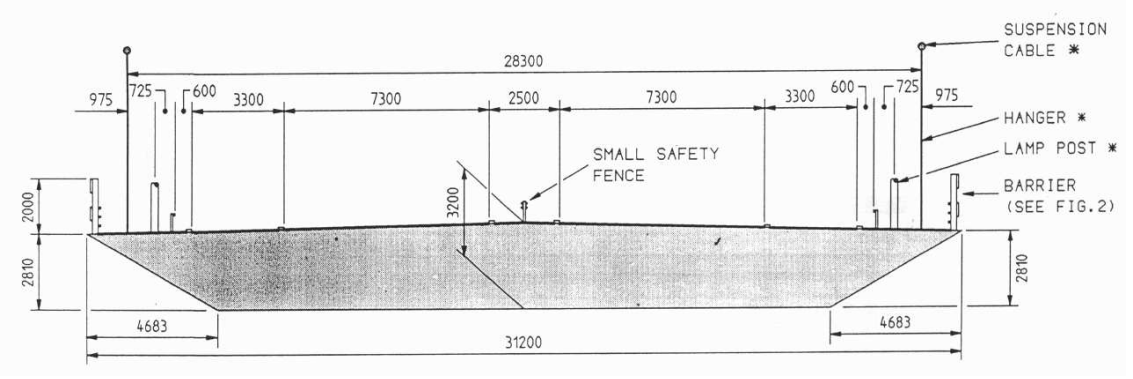

Figure 1. General arrangement of deck section

\section{BRIDGE DECK MODEL}

All the computational experiments contained in this paper were performed in order to compare with the experimental tests undertaken by BMT Fluid Mechanics Ltd. The experiments were carried out to predict the critical wind speed for the onset of instability for the Second Forth 


\begin{tabular}{|c|c|}
\hline Overall Width $(D)$ & $31.2 \mathrm{~m}$ \\
\hline Maximum Depth & $3.2 \mathrm{~m}$ \\
\hline Polar Mass Moment of Inertia $(I)$ & $2.13 \times 10^{6} \mathrm{kgm}^{2}$ \\
\hline Mass $(M)$ & $17.3 \times 10^{3} \frac{\mathrm{kg}}{\mathrm{m}}$ \\
\hline Natural Torsion Frequency $\left(f_{\theta}\right)$ & $0.4 \mathrm{~Hz}$ \\
\hline Natural Heaving Frequency $\left(f_{y}\right)$ & $0.174 \mathrm{~Hz}$ \\
\hline Centre of Mass & $2.09 \mathrm{~m}$ above soffit \\
\hline Centre of Shear & $1.46 \mathrm{~m}$ above soffit \\
\hline
\end{tabular}

Table I. Full scale properties of the Forth Road Bridge deck

Road Bridge design shown in figure 1. The main full-scale properties of the deck are shown in table I. Table II lists the structural non-dimensional parameters that have to be consistent with the full scale bridge deck in order to ensure dynamic similarity, these include the logarithmic decrement for both heave, $\delta_{y}$, and rotation, $\delta_{\theta}$. In addition to the structural parameters the aerodynamic parameters of reduced velocity and Reynolds number must also be consistent.

\begin{tabular}{|c|c|c|c|c|}
\hline$\frac{M}{\rho D^{2}}$ & $\frac{I}{\rho D^{4}}$ & $\delta_{\theta}$ & $\delta_{y}$ & $\frac{f_{\theta}}{f_{y}}$ \\
\hline 14.508 & 1.835 & 0.009 & 0.019 & 2.300 \\
\hline
\end{tabular}

Table II. Non-dimensional properties of the full-scale bridge deck

In wind tunnel tests the 1:65 model was free to rotate and heave. The critical wind speed was evaluated by measuring the root mean squared (rms) of the displacement for both rotation and translation and was assumed to occur at the point of rapid response of displacement for increasing reduced velocity. The experiment predicted a critical reduced velocity of $6.35 \pm 0.6$.

The numerical results are compared to experimental data for a case where the wind tunnel model is at zero incidence and only the central barrier remains, i.e. the wind barriers and parapets are removed. The central barriers are not contained within our computational domain, a portion of which can be seen in figure 2 . The computational mesh used in all the numerical calculations is made up of 1789 elements. All simulations in this paper were performed using a polynomial order of $p=5$, which implies 15 local degrees of freedom for each element.

\section{PREDICTION OF FLUTTER INSTABILITY}

\subsection{Linear expansion of aerodynamic forces using aerodynamic derivatives}

A widely used method to determine the critical reduced velocity for the onset of flutter instability of an elastically mounted body is to represent the lift and moment linearly 


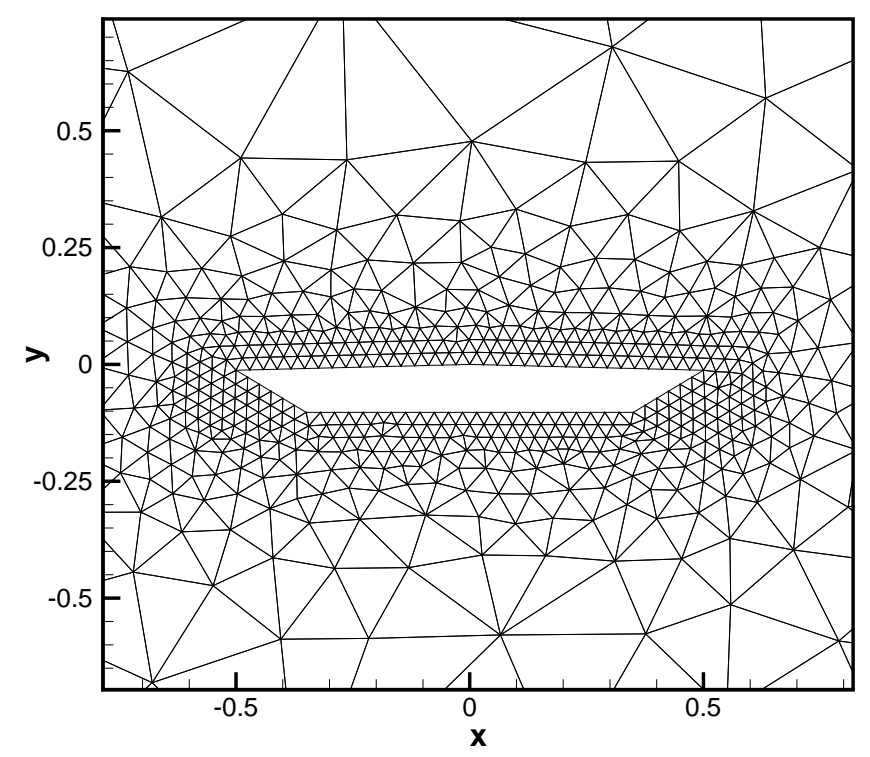

Figure 2. Close up of the computational mesh around the Forth Road Bridge deck.

$[15,16]$. The force and moment is presumed to be dependent on the angular and translational displacement and velocity, and also the aerodynamic derivatives associated with the body. These aerodynamic derivatives are proportional to the variation of lift and moment with displacement and velocity. The aerodynamic derivatives can be evaluated analytically by assuming a flat plate analogy or numerically by performing a series experiments where the motion of the bridge is prescribed.

Linear expressions for the lift, $F_{y}$, and moment, $M_{\theta}$, of a heaving and rotating body of length $D$ are given in terms of the non-dimensional aerodynamic derivatives $H_{i}^{*}$ and $A_{i}^{*}(i=1,2,3,4)$ by Simiu \& Scanlan [15] as,

$$
\begin{aligned}
F_{y} & =K H_{1}^{*} \dot{y}+K H_{2}^{*} \dot{\theta}+K^{2} H_{3}^{*} \theta+K^{2} H_{4}^{*} y, \\
M_{\theta} & =K A_{1}^{*} \dot{y}+K A_{2}^{*} \dot{\theta}+K^{2} A_{3}^{*} \theta+K^{2} A_{4}^{*} y,
\end{aligned}
$$

where $K=\frac{D \omega}{U}$ and represents the reduced frequency of the motion. Therefore the full physical system of the oscillating body can be expressed in terms of the linear expressions for the structural motion of the body as well as the lift and moment due to the aerodynamic forces on the body. The resulting equations of motion for heave and rotation are,

$$
\begin{aligned}
\ddot{y}+2 \zeta_{y} K_{y} \dot{y}+K_{y}^{2} y & =\frac{1}{n_{y}}\left[K H_{1}^{*} \dot{y}+K H_{2}^{*} \dot{\theta}+K^{2} H_{3}^{*} \theta+K^{2} H_{4}^{*} y\right], \\
\ddot{\theta}+2 \zeta_{\theta} K_{\theta} \dot{\theta}+K_{\theta}^{2} \theta & =\frac{1}{n_{\theta}}\left[K A_{1}^{*} \dot{y}+K A_{2}^{*} \dot{\theta}+K^{2} A_{3}^{*} \theta+K^{2} A_{4}^{*} y\right],
\end{aligned}
$$


where $K_{y}=\frac{D \omega_{y}}{U}$ and $K_{\theta}=\frac{D \omega_{\theta}}{U}$ and $\omega_{y}$ and $\omega_{\theta}$ represent the circular natural frequencies of the body for translational and rotational oscillations respectively. It is theorised that the oscillatory motion of the body is sinusoidal and the flutter instability occurs when the heave and rotational motion are of the same frequency $[15,16,17]$, therefore the translational and rotational displacements are represented as ,

$$
\begin{gathered}
h(t)=A_{y} e^{i K t}, \\
\theta(t)=A_{\theta} e^{i K t} .
\end{gathered}
$$

Substituting equations (19) and (20) into equations (17) and (18) results in the following relationships for the unknown $K$,

$$
\left[-K^{2}+2 \zeta_{y} K_{y} K i+K_{y}^{2}-\frac{1}{n_{y}} K^{2} H_{1}^{*} i-\frac{1}{n_{y}} K^{2} H_{4}^{*}\right] A_{y}-\left[\frac{1}{n_{y}} K^{2} H_{2}^{*} i+\frac{1}{n_{y}} K^{2} H_{3}^{*}\right] A_{\theta}=0
$$

and

$$
\left[-\frac{1}{n_{\theta}} K^{2} A_{1}^{*} i-\frac{1}{n_{\theta}} K^{2} A_{4}^{*}\right] A_{y}+\left[-K^{2}+2 \zeta_{\theta} K_{\theta} K i+K_{\theta}^{2}-\frac{1}{n_{\theta}} K^{2} A_{2}^{*} i-\frac{1}{n_{\theta}} K^{2} A_{3}^{*}\right] A_{\theta}=0 .
$$

Equations (21) and (22) can be represented in a matrix format as,

$$
\left[\begin{array}{ll}
A & B \\
C & D
\end{array}\right]\left[\begin{array}{l}
A_{y} \\
A_{\theta}
\end{array}\right]=\left[\begin{array}{l}
0 \\
0
\end{array}\right]
$$

where

$$
\begin{aligned}
A & =-K^{2}+2 \zeta_{y} K_{y} K i+K_{y}^{2}-\frac{1}{n_{y}} K^{2} H_{1}^{*} i-\frac{1}{n_{y}} K^{2} H_{4}^{*}, \\
B & =\frac{1}{n_{y}} K^{2} H_{2}^{*} i+\frac{1}{n_{y}} K^{2} H_{3}^{*} \\
C & =-\frac{1}{n_{\theta}} K^{2} A_{1}^{*} i-\frac{1}{n_{\theta}} K^{2} A_{4}^{*}, \\
D & =-K^{2}+2 \zeta_{\theta} K_{\theta} K i+K_{\theta}^{2}-\frac{1}{n_{\theta}} K^{2} A_{2}^{*} i-\frac{1}{n_{\theta}} K^{2} A_{3}^{*} .
\end{aligned}
$$

For the system to have a non-trivial solution the determinant of the matrix solution system (23) must be zero, i.e.

$$
A D-C B=0 .
$$

The solution is then evaluated to determine a value of $K$ which satisfies equation (28). $K$ is taken to be a complex number such that $K=K_{R}+i K_{I}$ where the real part represents the frequency of the oscillations and the imaginary part of $K$ dictates whether the solution is growing or decaying. A positive value of $K_{I}$ indicates a damped solution and a negative value specifies oscillations of increasing amplitude. Where $K_{I}$ is zero indicates the critical reduced velocity for the onset of flutter instability.

\subsection{Aerodynamic derivatives of a flat plate}

The solution of system (28) relies on the evaluation of accurate aerodynamic derivatives for the oscillating body being studied. Theodorsen [17] derived an analytical method where

Int. J. Numer. Meth. Fluids 2000; 00:1-6 


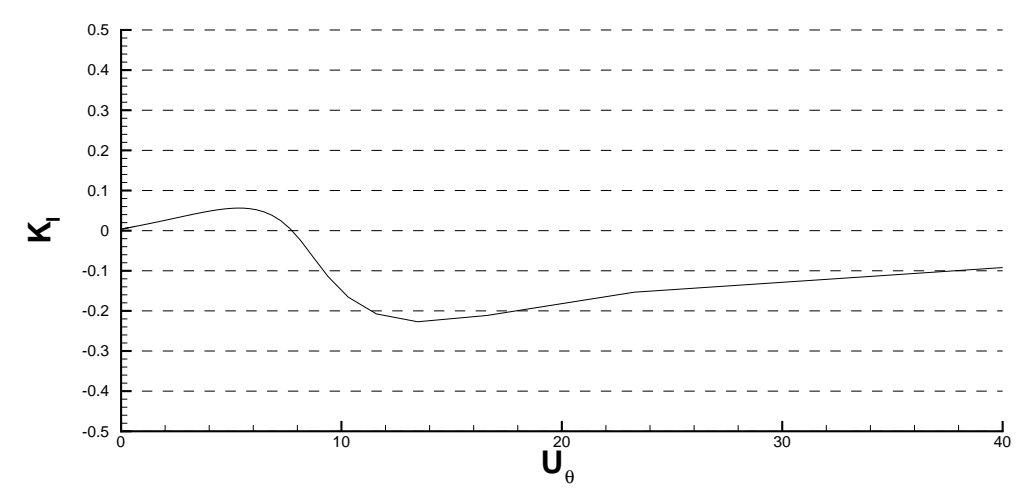

Figure 3. Imaginary part of $K$ indicating flutter limit using analytically derived aerodynamic derivatives for a flat plate [17]

the aerodynamic derivatives are determined by comparing the body with a flat plate in a potential flow. The flat plate has the same structural characteristics as the oscillating body and the aerodynamic derivatives are given in terms of the Theodorsen circulation function $C(k)=F(k)+i G(k)$ as,

$$
\begin{aligned}
H_{1}^{*}=-\frac{\pi F(k)}{2 k} \quad, \quad H_{2}^{*}=-\frac{\pi}{8 k}\left[1+F(k)+\frac{2 G(k)}{k}\right], \\
H_{3}^{*}=-\frac{\pi}{4 k^{2}}\left[F(k)-\frac{k G(k)}{2}\right] \quad, \quad H_{4}^{*}=\frac{\pi}{4}\left[1+\frac{2 G(k)}{k}\right], \\
A_{1}^{*}=\frac{\pi F(k)}{8 k} \quad, \quad A_{2}^{*}=-\frac{\pi}{32 k}\left[1-F(k)-\frac{2 G(k)}{k}\right], \\
A_{3}^{*}=\frac{\pi}{16 k^{2}}\left[F(k)-\frac{k G(k)}{2}\right] \quad, \quad A_{4}^{*}=-\frac{\pi}{8} \frac{G(k)}{k},
\end{aligned}
$$

where $F(k)$ and $G(k)$ are given by Bessel's functions of the first and second kind and of the zero and first order:

$$
\begin{gathered}
F(k)=\frac{J_{1}\left(J_{1}+Y_{0}\right)+Y_{1}\left(Y_{1}-J_{0}\right)}{\left(J_{1}+Y_{0}\right)^{2}+\left(Y_{1}-J_{0}\right)^{2}}, \\
G(k)=-\frac{Y_{1} Y_{0}+J_{1} J_{0}}{\left(J_{1}+Y_{0}\right)^{2}+\left(Y_{1}-J_{0}\right)^{2}},
\end{gathered}
$$

and $k$ is the reduced frequency based on the half-chord length, such that $k=\frac{\omega D}{2 U}$. The values of the aerodynamic derivatives are substituted into equation (28) and solved for varying values of $k$. When $K_{I}$ is zero the critical reduced velocity, $U_{c}$, can be found using the relationship;

$$
U_{c}=\frac{K_{R_{c}}}{k_{c}} \frac{\pi}{K_{\theta}}
$$

where $K_{R_{c}}$ and $k_{c}$ represent the critical values of $K_{R}$ and $k$ at the point $K_{I}=0$. 
Figure 3 shows the variation of $K_{I}$ for increasing reduced velocity. The critical reduced velocity can be found from the graph and is equal to $U_{c}=7.72$. In can be seen from the graph that as $U_{\theta} \rightarrow 0$ and therefore $U \rightarrow 0, K_{I} \rightarrow 0$ due to the absence of a free stream to excite the bridge deck section. Similarly as $U_{\theta} \rightarrow \infty$ and $K_{\theta} \rightarrow 0$ the bridge deck will not oscillate as it has no natural frequency to excite.

\subsection{Numerically derived aerodynamic derivatives}

For any single geometry the aerodynamic derivatives are dependent on the reduced velocity and can be numerically or experimentally evaluated by prescribing the oscillation of a body to be purely translational or purely rotational. Equations (15) and (16) are therefore reduced to

$$
\begin{aligned}
F_{y} & =K H_{1}^{*} \dot{y}+K^{2} H_{4}^{*} y, \\
M_{\theta} & =K A_{1}^{*} \dot{y}+K^{2} A_{4}^{*} y,
\end{aligned}
$$

for purely heave motion and

$$
\begin{aligned}
F_{y} & =K H_{2}^{*} \dot{\theta}+K^{2} H_{3}^{*} \theta, \\
M_{\theta} & =K A_{3}^{*} \dot{\theta}+K^{2} A_{3}^{*} \theta,
\end{aligned}
$$

for purely rotational motion. The aerodynamic derivatives, $H_{1}^{*}, H_{4}^{*}, A_{1}^{*}$ and $A_{4}^{*}$ can therefore be solved for using the matrix system below,

$$
\left[\begin{array}{cc}
K \dot{y}_{1} & K^{2} y_{1} \\
K \dot{y}_{2} & K^{2} y_{2} \\
\vdots & \vdots \\
K \dot{y}_{j} & K^{2} y_{j} \\
\vdots & \vdots \\
K \dot{y}_{n s} & K^{2} y_{n s}
\end{array}\right]\left[\begin{array}{c}
H_{1}^{*} \\
H_{4}^{*}
\end{array}\right]=\left[\begin{array}{c}
F_{y_{1}} \\
F_{y_{2}} \\
\vdots \\
F_{y_{j}} \\
\vdots \\
F_{y_{n s}}
\end{array}\right]
$$

and

$$
\left[\begin{array}{cc}
K \dot{y}_{1} & K^{2} y_{1} \\
K \dot{y}_{2} & K^{2} y_{2} \\
\vdots & \vdots \\
K \dot{y}_{j} & K^{2} y_{j} \\
\vdots & \vdots \\
K \dot{y}_{n s} & K^{2} y_{n s}
\end{array}\right]\left[\begin{array}{c}
A_{1}^{*} \\
A_{4}^{*}
\end{array}\right]=\left[\begin{array}{c}
M_{\theta_{1}} \\
M_{\theta_{2}} \\
\vdots \\
M_{\theta_{j}} \\
\vdots \\
M_{\theta_{n s}}
\end{array}\right]
$$

where $1 \leq j \leq n s$ and $n s$ denotes the number of sampling points in time. The matrix systems in equations (40) and (41) are solved using a least squares technique and the remaining aerodynamic derivatives are evaluated in an analogous manner using the corresponding results from purely rotational oscillations.

The bridge is forced to oscillate at various reduced frequencies to evaluate the aerodynamic derivatives at these frequencies. The critical reduced velocity can then be solved in an identical manner as discussed in section 4.2. Figure 4 shows the variations of $K_{I}$ with reduced velocity, where a reduced velocity of 6.21 results in a zero value of $K_{I}$.

Int. J. Numer. Meth. Fluids 2000; 00:1-6 


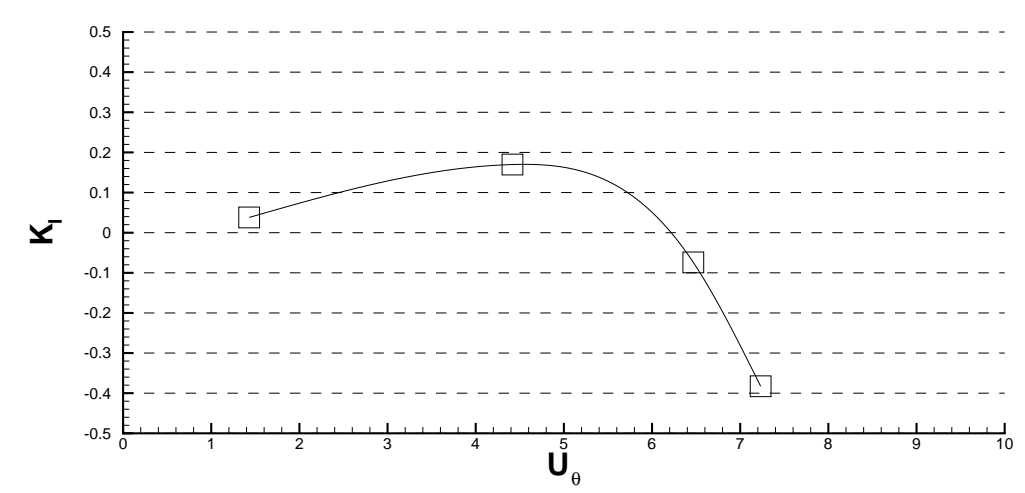

Figure 4. Imaginary part of $K$ indicating flutter limit using numerically derived aerodynamic derivatives

\subsection{Linear quasi-steady formulation}

The use of quasi-steady analysis has long been applied to evaluate the potential of a body to undergo heave galloping $[18,19]$. Quasi-steady analysis has been successful in this area as the time period of the motion of the body is far longer than the time period of the fluctuations of the flow and therefore it is the time averaged properties of the flow which significantly influence the mechanics of the body.

The quasi-steady formulation is dependent on the evaluation of the variation of lift and moment with displacement, whilst the method outlined in section 4.3 used the variation of lift and moment with displacement and velocity. Therefore, the main difference between this technique and the previous one is that the determination of the critical reduced velocity is solely dependent on static calculations when considering the quasi-steady technique, whereas the evaluation of the aerodynamic derivatives requires dynamic calculations.

Using quasi-steady principles the effective angle of attack, $\alpha$, is related to the vertical velocity of the body:

$$
\alpha=-\dot{y} .
$$

Quasi-steady theory is less widely applied to the rotational motion of a body. Though it has been shown $[18,19]$ that the effective angle of incidence for purely rotational oscillations is proportional to the actual angle of attack and the angular velocity of the body:

$$
\alpha=\theta-R \dot{\theta},
$$

where $R$ is chosen to the half-chord length non-dimensionalised by $D[18,20]$. If the bridge is undergoing both translational and rotational motion the effective, linearised angle of attack is the combination of equations (42) and (43);

$$
\alpha=\theta-R \dot{\theta}-\dot{y} .
$$

The motion of the bridge can be related to the aerodynamic force and moment, represented 
as the force coefficients, $F_{y}$ and $M_{\theta}$ by,

$$
\begin{aligned}
& \ddot{y}+2 \zeta_{y} K_{y} \dot{y}+K_{y}^{2} y=\frac{F_{y}}{n_{y}}, \\
& \ddot{\theta}+2 \zeta_{\theta} K_{\theta} \dot{\theta}+K_{\theta}^{2} \theta=\frac{M_{\theta}}{n_{\theta}} .
\end{aligned}
$$

Therefore, by substituting equation (44) into equation (46) the force coefficients can be represented as,

$$
\begin{aligned}
F_{y}(\alpha) & =F_{y}(\alpha=0)+\left.\frac{d F_{y}}{d \alpha}\right|_{\alpha=0} \underset{\alpha}{\alpha}, \\
& =F_{y}(\alpha=0)+\left.\frac{d F_{y}}{d \alpha}\right|_{\alpha=0}(\theta-R \dot{\theta}-\dot{y}), \\
M_{\theta}(\alpha) & =M_{\theta}(\alpha=0)+\left.\frac{d M_{\theta}}{d \alpha}\right|_{\alpha=0} ^{\alpha}, \\
& =M_{\theta}(\alpha=0)+\left.\frac{d M_{\theta}}{d \alpha}\right|_{\alpha=0}(\theta-R \dot{\theta}-\dot{y}) .
\end{aligned}
$$

The motion of the structure is therefore governed by the following linear equations,

$$
\begin{aligned}
& \ddot{y}+2 \zeta_{y} K_{y} \dot{y}+K_{y}^{2} y=\frac{1}{n_{y}} \frac{d F_{y}}{d \alpha}(\theta-R \dot{\theta}-\dot{y}), \\
& \ddot{\theta}+2 \zeta_{\theta} K_{\theta} \dot{\theta}+K_{\theta}^{2} \theta=\frac{1}{n_{\theta}} \frac{d M_{\theta}}{d \alpha}(\theta-R \dot{\theta}-\dot{y}),
\end{aligned}
$$

where the values of $F_{y}(\alpha=0)$ and $M_{\theta}(\alpha=0)$ are omitted as they cause static displacements and do not influence the flutter stability of the structure. The system is dependent on the variation of $F_{y}$ and $M_{\theta}$ with $\alpha$. This variation for the Forth Road Bridge deck design is graphically shown in figure 5 . These results were evaluated at $R e=10000$ under twodimensional assumptions and $\left.\frac{d F_{y}}{d \alpha}\right|_{\alpha=0}=5.34$ and $\left.\frac{d M_{\theta}}{d \alpha}\right|_{\alpha=0}=1.40$.

The onset of flutter is once again presumed to occur when the frequency of the rotational and translational motion coincides. Solutions of the form,

$$
y=A_{y} e^{i K t}, \quad \theta=A_{\theta} e^{i K t},
$$

are taken where $K=\frac{\omega D}{U}=K_{R}+K_{I} i$. The variable $\omega$ is complex with its imaginary part representing the frequency of the oscillations and its real part dictating the damping of the system. By substituting the above solutions for $y$ and $\theta$ and collecting $A_{y}$ and $A_{\theta}$ terms we have,

$$
\begin{gathered}
{\left[-K^{2}+K_{y}^{2}+2 \zeta_{y} K_{y} K i+\frac{1}{2 n_{y}} \frac{d C_{y}}{d \alpha} K i\right] A_{y}+\left[-\frac{1}{2 n_{y}} \frac{d C_{y}}{d \alpha}+\frac{1}{2 n_{y}} \frac{d C_{y}}{d \alpha} R K i\right] A_{\theta}} \\
{\left[\frac{1}{2 n_{\theta}} \frac{d C_{m}}{d \alpha} K i\right] A_{y}+\left[-K^{2}+K_{\theta}^{2}-\frac{1}{2 n_{\theta}} \frac{d C_{m}}{d \alpha}++2 \zeta_{\theta} K_{\theta} K i+\frac{1}{2 n_{\theta}} \frac{d C_{m}}{d \alpha} R K i\right] A_{\theta} .}
\end{gathered}
$$




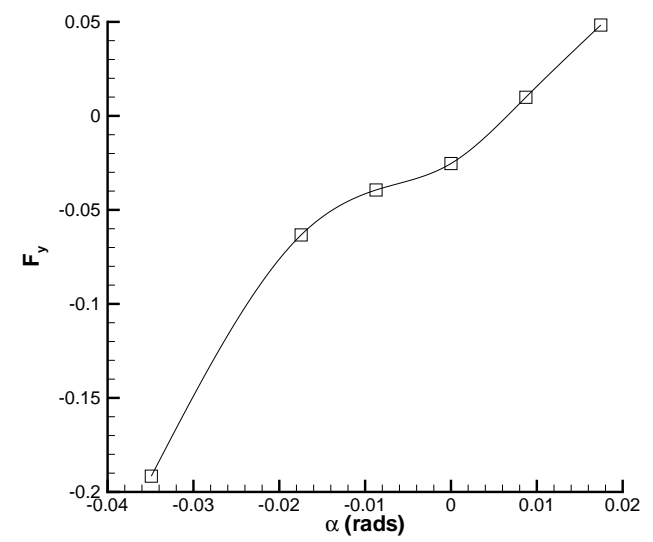

(a) $F_{y}$ against $\alpha$

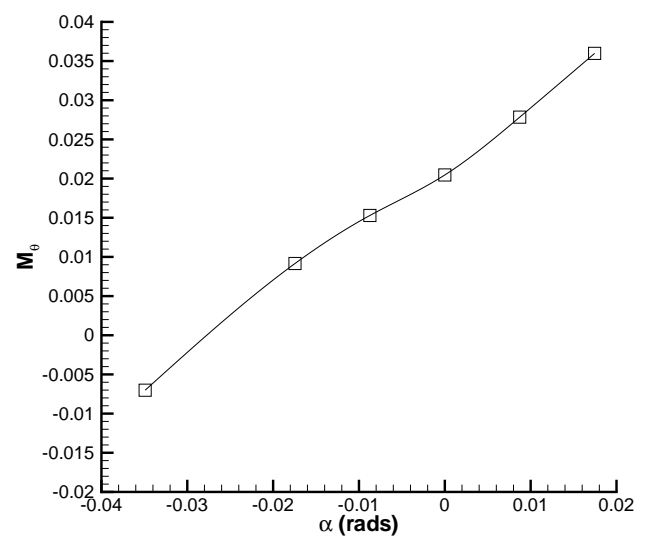

(b) $M_{\theta}$ against $\alpha$

Figure 5. Variation of $F_{y}$ and $M_{\theta}$ with $\alpha$ for the Forth Bridge Design

which are again represented as,

$$
\left[\begin{array}{ll}
A & B \\
C & D
\end{array}\right]\left[\begin{array}{l}
A_{y} \\
A_{\theta}
\end{array}\right]=\left[\begin{array}{l}
0 \\
0
\end{array}\right]
$$

and for the system to have a non-trivial solution

$$
A B-C D=0 .
$$

As before the value of $K_{I}$ indicates whether a solution is growing or decaying and a positive value of $K_{I}$ indicates a damped solution and a negative value indicates a growing solution. The variation of $K_{I}$ with reduced velocity $U_{\theta}=\frac{U}{f_{\theta} D}$ is shown in figure 6 . These results predict the critical reduced velocity for the Forth Road Bridge design as 6.58. The variation of $K_{I}$ with increasing $U_{\theta}$ is as expected, that is $K_{I} \rightarrow 0$ as $U_{\theta} \rightarrow \infty$. As $U_{\theta} \rightarrow 0, K_{I}$ does not converge to zero, though it must be considered that the amplitudes of the oscillation, $A_{\theta}$ and $A_{y}$ can take a zero value for $U_{\theta}=0$

\subsection{Freely oscillating bridge deck}

The critical reduced velocity can be evaluated by performing a series of numerical experiments where the bridge deck is allowed to oscillate under the specified governing structural parameters. The reduced velocity is incrementally increased and the point at which the translational or rotational amplitude of the oscillations steadily increases is taken as the critical reduced velocity for the onset of flutter instability.

Time histories for the rotational and heaving displacements for increasing reduced velocity can be seen in figures 7 and 8 respectively. As can be seen from the graphs the magnitude of the oscillations quickly decrease for $U_{\theta}=2.5$ for both rotation and heave. At larger reduced 


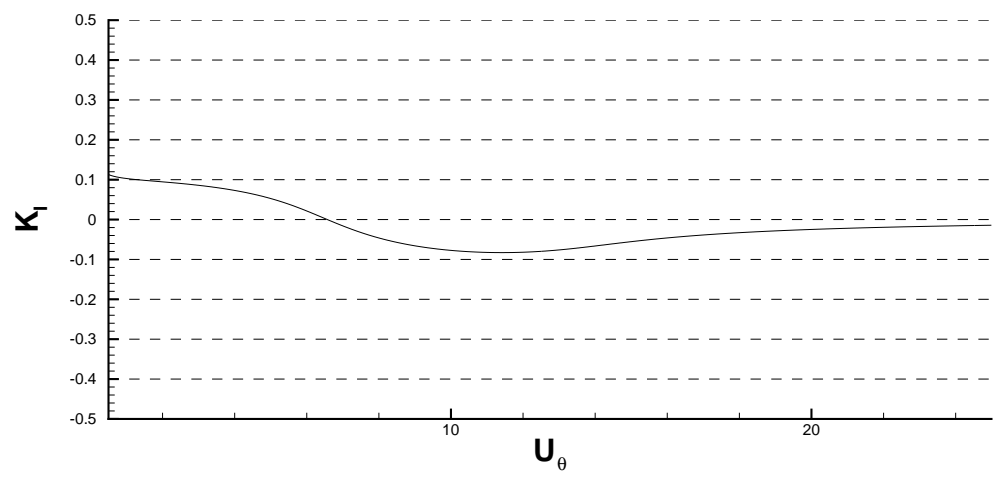

Figure 6. Imaginary part of $K$ indicating flutter limit using quasi-steady analysis

velocities the oscillations maintain their magnitude, the value of which is dependent on the reduced velocity.

The variation of the frequencies of the rotational and heave motion for increasing reduced velocity are shown in figure 9 . These results are in agreement with the assumption made by the aerodynamic derivatives approach (equations (19) and (20)) and the quasi-steady technique (equation (53) that at the point of flutter instability the frequencies of the translational and rotational motion is identical.

Figure 10 shows the instantaneous flow field and pressure distribution around the structure for a complete rotational oscillation. The contours shown represent vorticity where black indicates a high value of vorticity and grey a low value of vorticity. A similar scheme is used for the pressure distribution where black shading indicates a positive pressure and grey shading a negative pressure. The flow separates from the upper leading edge resulting in an area of low pressure. As the bridge deck oscillates the point where this shear layer re-attaches constantly changes leading to pressure fluctuations which drive the high amplitude oscillations shown in figure $7(\mathrm{c})$ and $8(\mathrm{c})$.

Figures 11 and 12 show the computational and experimental rms values of rotational and translational displacement respectively. The two sets of data are in reasonable agreement, particularly when the disparity in Reynolds number between the numerical simulations and experiments is considered. The computations were performed for reduced velocities, $U_{\theta}$, ranging from 2.5 to 7 and Reynolds numbers between 4167 and 11667, whilst the experimental Reynolds number over the same range would have been of the order of $1 \times 10^{5}$ and $1 \times 10^{6}$. The rms for heaving and rotation appears to be over predicted for $U_{\theta}$ equal to 5 and 6 and under predicted for $U_{\theta}=7$. This could be a consequence of three-dimensional effects captured in the experiments and not included in the numerical simulations. The experimental set-up would have included three dimensional effects which the numerical calculations have omitted. At low oscillation amplitudes we would expect the vortical structures produced experimentally to have a finite correlation length and corresponding decorrellation of the forces and moments along the length of the bridge deck section. It has been shown for circular cylinders that when the oscillations increase in size the vortical structures become homogeneous along the 


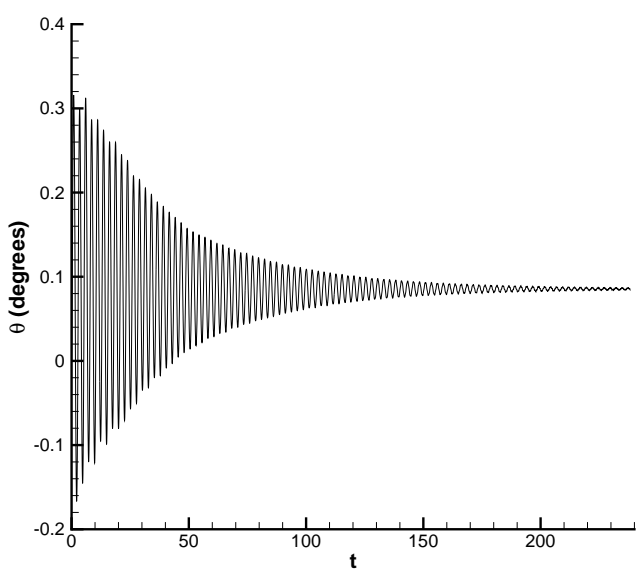

(a)

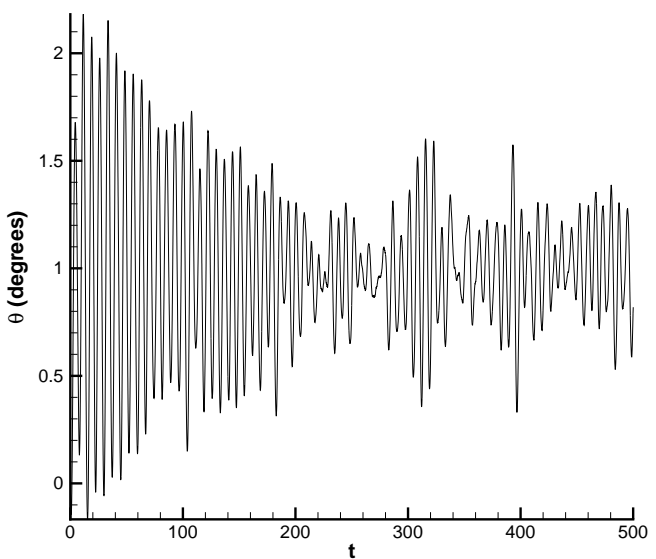

(c)

Figure 7. Rotational displacement of Forth Road Bridge Deck (a) $U_{\theta}=2.5$. (b) $U_{\theta}=5$. (c) $U_{\theta}=6$. (d) $U_{\theta}=7$.

length of the body $[21,22]$ and we therefore make the conjecture that this phenomenon also occurs during the experiments discussed in this paper. The enforced correlation of the two dimensional computations would therefore be expected to produce a larger amplitude than the experimental results.

The experimental results predicted the critical wind speed for the onset of instability to be $6.35 \pm 0.6$, whilst the numerical data predicts the onset of instability as between 6 and 7 .

\section{COMPARISON AND CONCLUSION}

The predicted critical wind velocities, $U_{c}$, are summarised below:

- Flat plate analogy: 7.72 ;

Copyright (c) 2000 John Wiley \& Sons, Ltd.

Int. J. Numer. Meth. Fluids 2000; 00:1-6

(b)

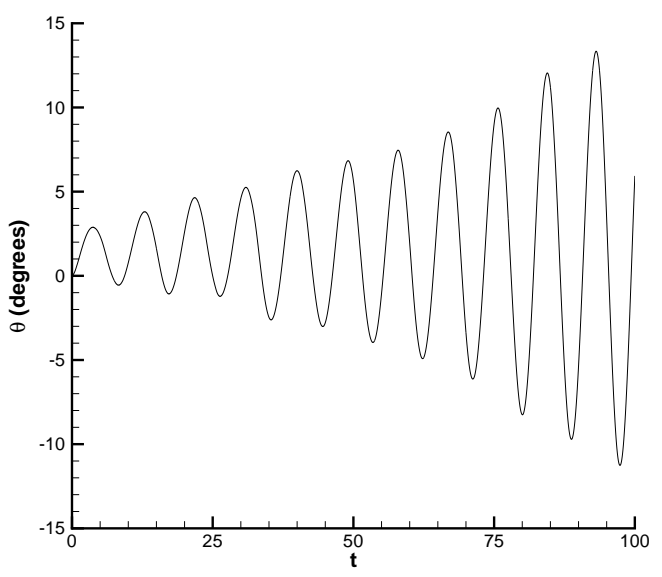

(d)

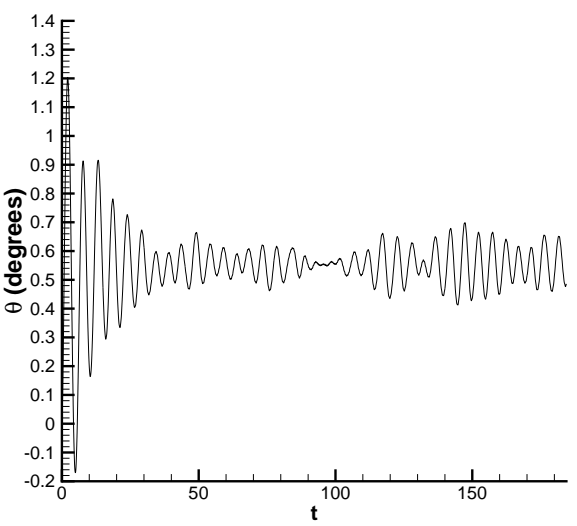




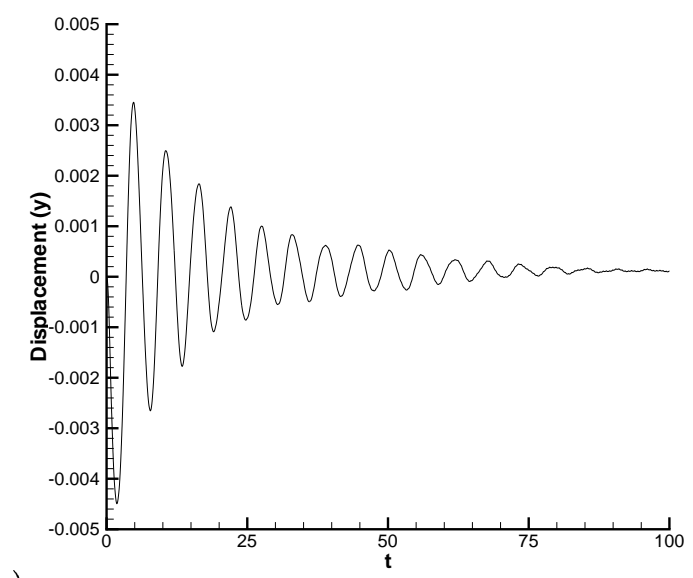

(a)

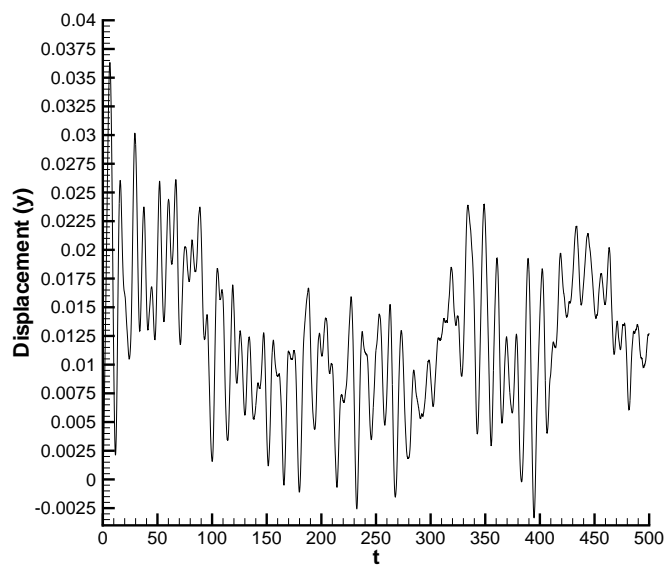

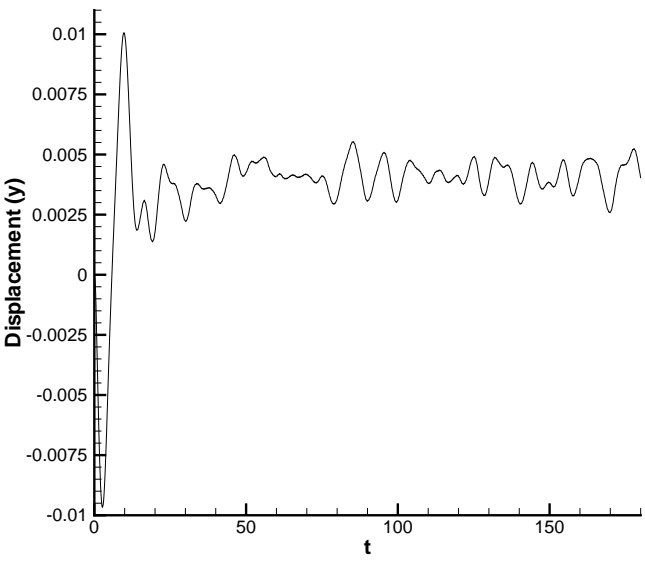

(b)

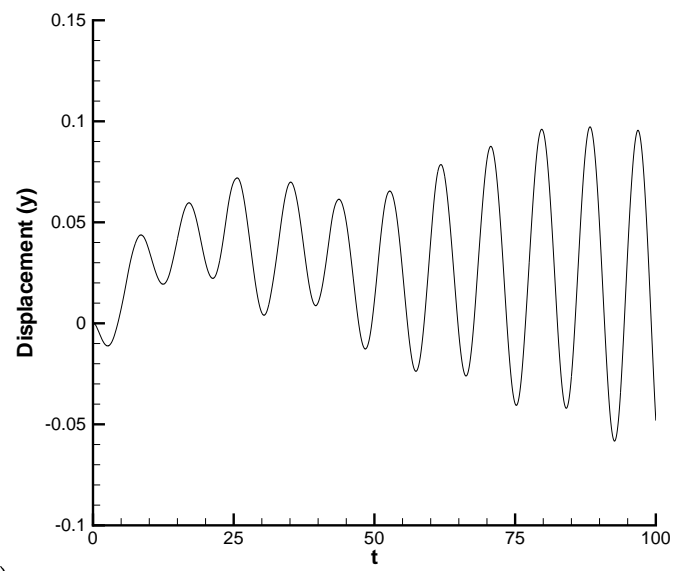

(d)

Figure 8. Heaving displacement of Forth Road Bridge Deck (a) $U_{\theta}=2.5$. (b) $U_{\theta}=5$. (c) $U_{\theta}=6$. (d) $U_{\theta}=7$.

- Numerically evaluated aerodynamic derivatives: 6.21 ;

- Linear quasi-steady formulation: 6.58 ;

- Numerical freely oscillating bridge deck: $6.5 \pm 0.5$;

- Experimental freely oscillating bridge deck: $6.35 \pm 0.6$.

All the analytical and numerical predictions compare favourably with the experimental result. The flat plate analogy produces the worst estimate supporting the observation that leading edge effects are important when evaluating flutter instabilities. As the flat plate analogy method requires no knowledge of the aerodynamic properties of the bridge deck and is near instantaneous to compute, it can still be regarded as an effective and economical tool to predict bridge deck flutter instabilities.

The agreement between the numerical and experimental oscillating bridge deck is particularly encouraging and evidence that the numerical algorithm is effective and could

Int. J. Numer. Meth. Fluids 2000; 00:1-6 


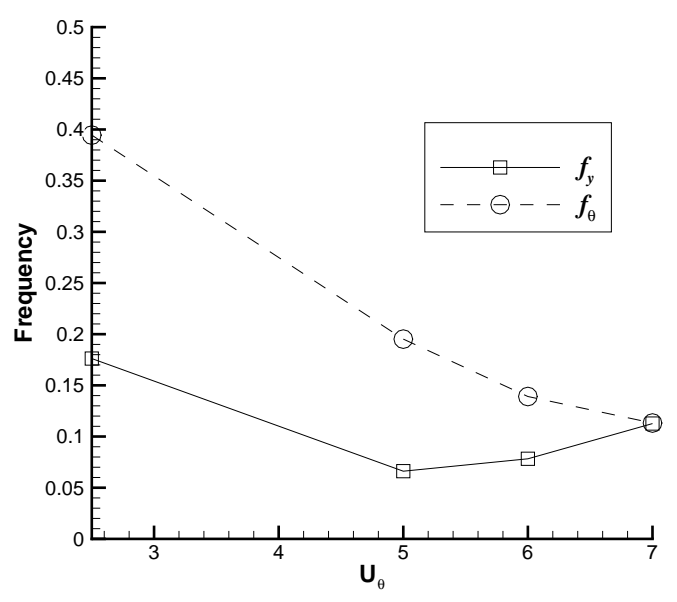

Figure 9. Variation of rotational, $f_{\theta}$, and heave, $f_{y}$, frequencies against reduced velocity

be utilised to investigate bridge designs and their sensitivity to leading edge modifications.

However, the role of three dimensional effects and Reynolds number scaling is still an open question. The geometry considered here has a sharp leading edge which is less likely to be as sensitive to Reynolds number and three dimensional effects as a rounded leading edge.

\section{Acknowledgments}

The work is support by EPSRC under grant GL/L48096. The authors would like to acknowledge the support and advice of Simon Coleman of BMT Fluid Mechanics Ltd and Brian Smith and David MacKenzie of Flint and Neill Partnership.

\section{REFERENCES}

1. Nomura, T. Finite element analysis of vortex-induced vibrations of bluff cylinders. Journal of Wind Engineering and Industrial Aerodynamics 1993; 46:587-594.

2. Wei R, Sekine A, Shimura M. Numerical analysis of $2 \mathrm{~d}$ vortex-induced oscillations of a circular cylinder. International Journal for Numerical Methods in Fluid 1995; 21:993-1005.

3. Frandsen JB, McRobie A. Finite element simulation of wind-induced bridge motion. In Computational Methods for Fluid Structure Interaction, T. Kvamsdal et al. (eds). Tapir Forlag: Trondheim, Norway, 1997.

4. Schulz K, Kallinderis Y. Unsteady flow structure interaction for incompressible flows using deformable hybrid grids. Journal of Computational Physics,1998; 143:569-597.

5. Walther JH, Larsen A. A two dimensional discrete vortex method for bridge aerodynamics applications. Forth European Computational Fluid Dynamics Conference, Athens, Greece; 1998.

6. Walther JH. Discrete Vortex Method for two-dimensional flow past bodies of arbitrary shape undergoing prescribed rotary and translatory motion. PhD Thesis, The Technical University of Denmark,Department of Fluid Mechanics, Lyngby, Denmark; 1994.

7. Newman D Karniadakis GE. A direct numerical simulation study of flow past a freely vibrating cable. Journal of Fluid Mechanics, 1998; 344:95-136.

Int. J. Numer. Meth. Fluids 2000; 00:1-6 
(a)
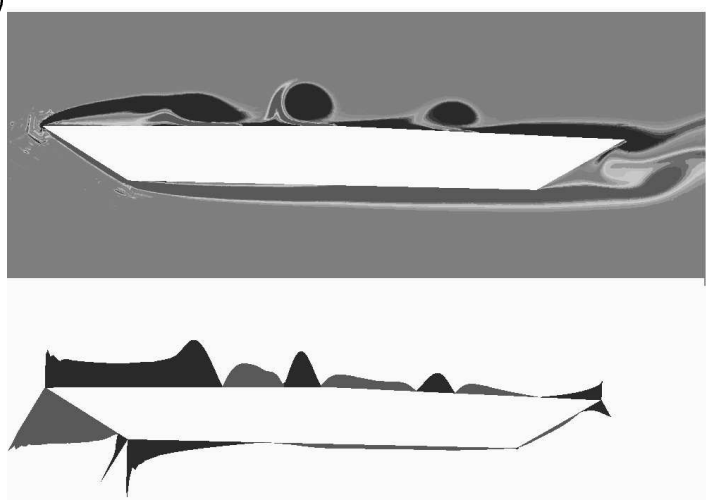

(c)

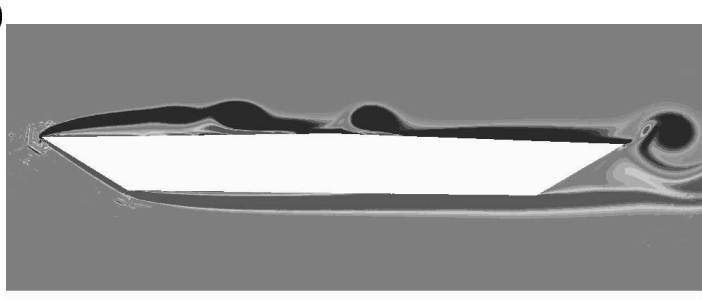

(d)
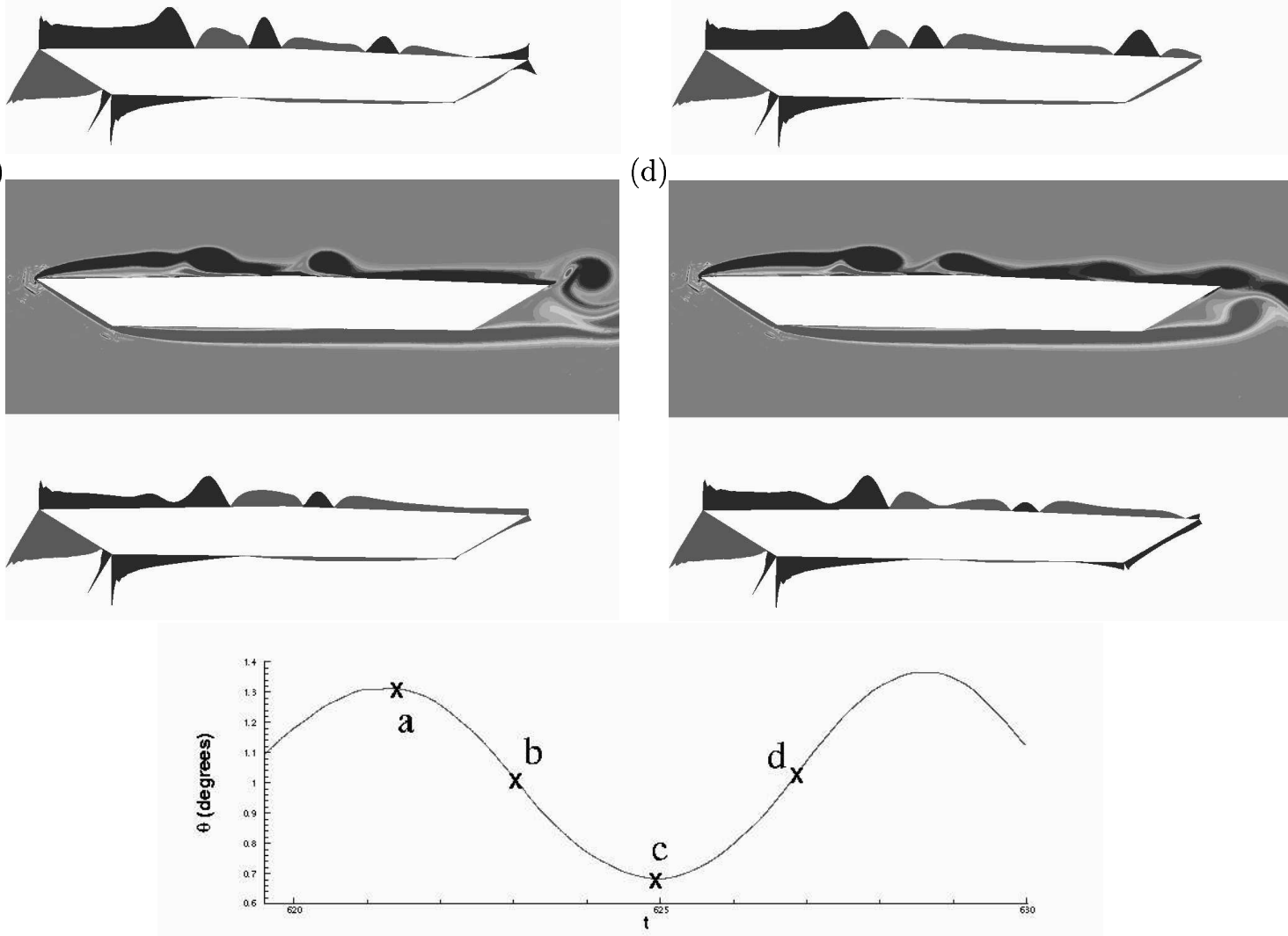

Figure 10. Flow field around the Forth Road Bridge Deck for $U_{\theta}=6$ over one rotational cycle

8. Anagnostopoulos P. Numerical investigation of response and wake characteristics of a vortex-excited cylinder in a uniform stream. Journal of Fluids and Structures, 1994; 8:367-390.

9. Piperno S. Numerical simulation of aeroelastic instabilities of elementary bridge decks. Rapport de recherche, $1998 ; n^{0} 3549$.

10. Li L, Sherwin SJ, Bearman PW. Numerical simulation of fluid/structure interacting problems using spectral element methods. International Journal of Numerical Methods in Fluids, 2002; 38: 187-206.

11. Steinman DB, Watson SR. Bridges and their builders. Dover, New York, 1957.

12. Scanlan RH. On the state of stability considerations for suspended-span bridges under wind. In Proceedings IUTAM-IAHR Symposium Karlsruhe, Germany, 1979: 595-618.

Copyright (C) 2000 John Wiley \& Sons, Ltd.

Int. J. Numer. Meth. Fluids 2000; 00:1-6

(b)

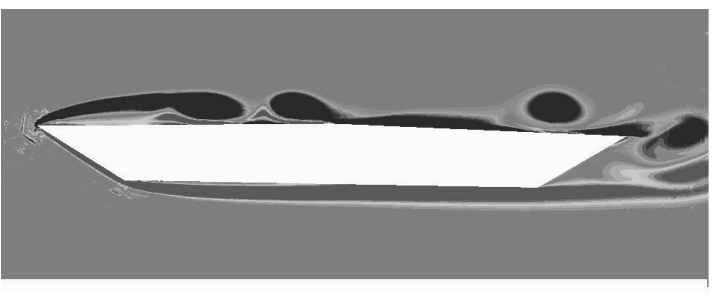




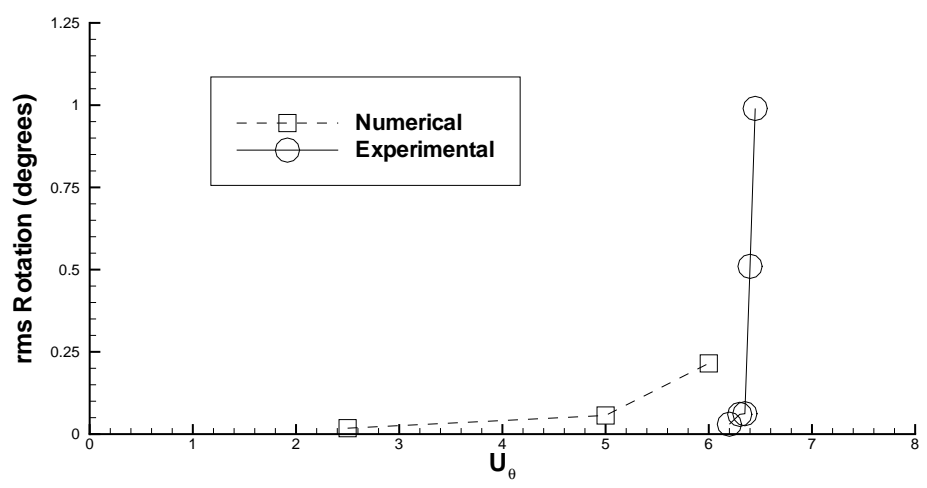

Figure 11. root mean square values of rotational displacement

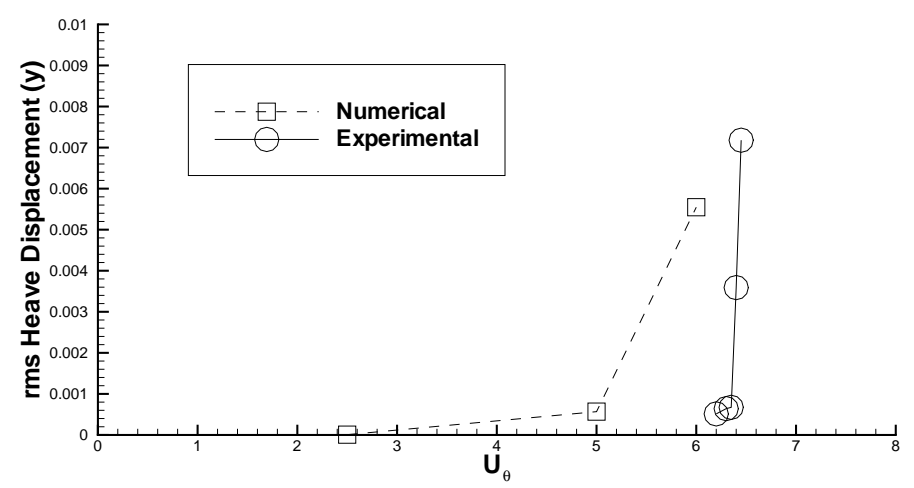

Figure 12. root mean square values of heaving displacement

13. Scanlan RH, Tomko JJ. Airfoil and bridge deck flutter derivatives, Journal of the Engineering Mechanics Division, ASCE, 1971; 97(EM6):1717-1737.

14. Nomura T, Hughes TJR. An arbitrary Lagrangian-Eulerian finite element method for interaction of fluid and a rigid body. Computer Methods in Applied Mechanics and Engineering, 1992; 95:115-138.

15. Simiu E, Scanlan RH. Wind effects on structures (3rd edn). John Wiley \& Sons, New York, 1996.

16. Dyrbye C, Hansen SO. Wind loads on structures. John Wiley \& Sons, New York,1997.

17. Theodorsen T. General theory of aerodynamic instability and the mechanism of flutter,, 1935; TR 496, NACA.

18. Blevins RD. Flow-induced vibration, New York: Van Nostrand Reinhold, 1990.

19. Robertson I, Li L, Sherwin SJ, Bearman PW. A numerical study of rotational and translational galloping instabilities, submitted to Journal of Fluids and Structures,, 2001.

20. Nakamura Y, Mizota T. Torsional Flutter of Rectangular Prisms, J. Eng. Mech. Div., Am. Soc. Civil Engrs., 1975; 101, EM 2: 125-142.

21. Kozakiewicz A, Sumer BM, Fredsoe J. Spanwise correlation on a vibrating cylinder near a wall in oscillatory flows, J. Fluids and Structures, 1992; 6: 371-392.

22. Sumer BM, Fredsoe J, Jensen K. A note on spanwise correlations on a freely vibrating cylinder in oscillatory flow, J. Fluids and Structures, 1994; 8: 231-238. 